\title{
Genomic analysis of carbapenemase-encoding plasmids from Klebsiella pneumoniae across Europe highlights three major patterns of dissemination
}

\section{Affiliations:}

${ }^{1}$ Centre for Genomic Pathogen Surveillance, Wellcome Genome Campus, Hinxton, Cambridge, CB10 1SA, United Kingdom

${ }^{2}$ Institute for Infection Prevention and Hospital Epidemiology, Medical Centre University of Freiburg, Faculty of Medicine, University of Freiburg, Breisacherstr 115b, 79106 Freiburg, Germany

${ }^{3}$ Modernizing Medical Microbiology Consortium, Nuffield Department of Clinical Medicine, John Radcliffe Hospital, Oxford University, Oxford, United Kingdom

${ }^{4}$ Department of Experimental and Clinical Medicine, University of Florence, Largo Brambilla 3, 50134 Florence, Italy

${ }^{5}$ Clinical Microbiology and Virology Unit, Florence Careggi University Hospital, Largo Brambilla 3, 50134 Florence, Italy

${ }^{6}$ Department of Veterinary Medicine, University of Cambridge, Cambridge, CB3 0ES, United Kingdom

${ }^{7} \mathrm{~A}$ full list of authors can be found at the end of the article

${ }^{8} \mathrm{~A}$ full list of authors can be found in the Supplementary Note

${ }^{9}$ Milner Centre for Evolution, Department of Biology and Biochemistry, University of Bath, Bath, UK

${ }^{10}$ Big Data Institute, Li Ka Shing Centre for Health Information and Discovery, Nuffield Department of Medicine, Oxford University, Oxford, UK

*These authors contributed equally.

Short title: Carbapenemase-encoding plasmids in Klebsiella pneumoniae

Corresponding authors: sophia.david@sanger.ac.uk and david.aanensen@bdi.ox.ac.uk

36 Keywords: Klebsiella pneumoniae, carbapenem resistance, carbapenemase genes, 


\section{Abstract}

The incidence of Klebsiella pneumoniae infections that are resistant to carbapenems, a last-line class of antibiotics, has been rapidly increasing. The primary mechanism of carbapenem resistance is production of carbapenemase enzymes, which are most frequently encoded on plasmids by blaoxA-48-like, blaviM, bla short-read sequence data, we previously analysed genomes of 1717 isolates from the K. pneumoniae species complex submitted during the European survey of carbapenemase-producing Enterobacteriaceae (EuSCAPE). Here, we investigated the diversity, prevalence and transmission dynamics of carbapenemase-encoding plasmids using long-read sequencing of representative isolates $(n=79)$ from this collection in combination with short-read data from all isolates. We highlight three major patterns by which carbapenemase genes have disseminated via plasmids. First, blaoxA-48-like genes have spread across diverse lineages primarily via a highly conserved, epidemic pOXA-48-like plasmid. Second, blavIM and blanDM genes have spread via transient associations of diverse plasmids with numerous lineages. Third, blakPC genes have transmitted predominantly by stable association with one clonal lineage (ST258/512) despite frequent mobilisation between pre-existing yet diverse plasmids within the lineage. Despite contrasts in these three modes of carbapenemase gene spread, which can be summarised as using one plasmid/multiple lineages, multiple plasmids/multiple lineages, and multiple plasmids/one lineage, all are underpinned by significant propagation along high-risk clonal lineages. 
The incidence of infections due to carbapenem-resistant Enterobacterales (CRE) is rapidly rising, posing a major challenge to public health globally (WHO, 2017). Indeed, carbapenem-resistant Klebsiella pneumoniae, the most clinically significant member of CRE, was recently highlighted as the fastest-growing resistance threat in Europe in terms of number of infections and attributable deaths (Cassini et al. 2019).

The largest subset of CRE, the carbapenemase-producing Enterobacterales (CPE), hydrolyse carbapenems and other beta-lactam antibiotics using diverse types of beta-lactamase enzymes called carbapenemases (David et al. 2019). Genes encoding these carbapenemases are usually located on plasmids which can transmit vertically along clonal lineages as well as horizontally between different strains and species (Mathers et al. 2011; Martin et al. 2017). Within plasmids, carbapenemase genes are also frequently associated with smaller mobile genetic elements such as transposons and mobile gene cassettes inserted into integrons, extending their recombinatory capability to multiple nested levels (Sheppard et al. 2016).

Next-generation sequencing using short-read technologies has vastly improved our ability to unravel the complexities of infectious disease epidemiology. In particular, it has enabled genomic surveillance of high-risk bacterial lineages including tracking of their geographical dissemination (Aanensen et al. 2016; Domman et al. 2017; Harris et al. 2018; David et al. 2019). These surveillance approaches typically use differences in a defined chromosomal region (the "core genome") that are determined by mapping sequence reads to a reference. However, advances in short-read sequencing have not enabled the same high-resolution tracking of plasmids since, typically being mosaic and recombinant, these usually require de novo assembly for accurate comparison. Unfortunately plasmid assemblies derived from short-read data are usually highly fragmented as a result of numerous repetitive elements (e.g. insertion sequences), and often cannot be distinguished from chromosomal sequences. Recently, these problems have been overcome by the advent of long-read sequencing, which now readily enables complete (or near-complete) and accurate resolution of plasmid sequences, particularly when the data are assembled together with short reads (Wick et al. 2017; George et al. 2017). This advance, coupled with

102 the decreasing costs of long-read sequencing, renders large-scale plasmid 
comparisons increasingly feasible and brings the benefits of the sequencing revolution to bear also on the molecular epidemiology of plasmids.

Despite the rapidly growing databases of carbapenemase-encoding plasmid sequences, no study has systematically analysed the diversity of these plasmids in clinical isolates across a large, unbiased and geographically diverse sample collection.

108 Previously, we analysed genomes of 1717 clinical isolates belonging to the $K$. 109 pneumoniae species complex sampled from 244 hospitals in 32 countries during the

110 European survey of CPE (EuSCAPE) (Grundmann et al. 2017; David et al. 2019). Six 111 hundred and seventy-eight (39.5\%) carried one or more of the blaoxA-48-like, blavIM, $112 b_{\text {blam }}$ and blaKPC carbapenemase genes. All carbapenemase-positive isolates 113 belonged to the $K$. pneumoniae sensu stricto species with the exception of one isolate 114 each from Klebsiella quasipneumoniae and Klebsiella variicola. Here we investigated 115 the diversity of carbapenemase-encoding plasmids amongst these isolates using 116 combined long- and short-read sequencing of selected representatives. Furthermore, 117 we explored the potential and limitations of using short-read sequence data obtained 118 from all isolates, together with reference plasmids obtained from representatives, to 119 assess the prevalence, distribution and transmission dynamics of carbapenemase120 encoding plasmids across the wider European population. These analyses revealed 121 three major patterns of plasmid transmission that have enabled widespread 122 dissemination of carbapenemase genes.

Results

Diversity of the genetic contexts of carbapenemase genes among $K$. pneumoniae

Of $1717 K$. pneumoniae species complex isolates submitted during the European Survey of CPE (EuSCAPE), we previously found that 249, 56, 79 and 312

133 Eighteen of these carried two genes. In this study, we first analysed the genetic contexts of these genes in short-read genome assemblies, considering this feature as 
carbapenemase genes were clustered into genetic context (GC) groups, based on the order and nucleotide similarity of genes flanking the carbapenemase gene (Supplementary Tables 1 and 2). Full contig sequences were used for this analysis. Contigs with fewer than four genes were excluded.

By this criterion, we identified 3,10,15 and 45 GC groups of isolates with different genetic contexts of blaoXA-48-like, blavIM, blanDM and blakPC genes, respectively (Table 1). Overall, 184/696 (26.4\%) of carbapenemase-carrying contigs could be unambiguously assigned to one of these groups. Assignment rates were higher for isolates carrying blaviM and blanDM, and lower for those carrying blaKPC and blaoXA-48like. In particular, only 4/249 (1.6\%) of blaoxA-48-like-carrying isolates could be assigned to a GC group due to the small size of the contigs, which typically carried only blaoxA48-like +/- lys $R$ genes.

We selected one isolate from each GC group for long-read sequencing, with the exception of one blaoxA-48-like group and two blakpc groups for which representative isolates were unavailable. Furthermore, since the above-described process resulted in selection of only two blaoxA-48-like-carrying isolates, we also selected an additional eight. These had blaoxA-48-like-carrying contigs with $\geq 4$ genes and, despite not clustering unambiguously into a single GC group, matched different combinations of other blaoxA-48-like-carrying contigs (Supplementary Table 2). They were therefore deemed the most likely to represent different plasmids amongst the remaining isolates. We also long-read sequenced another four blaoxA-48-like-carrying isolates which were positive for two carbapenemase genes and had been selected as representatives of blaKPC, blaviM or bla ${ }_{\mathrm{NDM}}$ GC groups. Furthermore, one isolate selected as a representative of a blakPC GC group also harboured blavim. Finally, we long-read sequenced two blaKPC-carrying isolates from the same GC group to investigate possible within-hospital plasmid transfer, since they were submitted from the same hospital but belonged to different sequence types (ST).

\section{Long-read sequencing of representative isolates revealed that most} carbapenemase genes were plasmid-borne

We assembled long-read sequencing data together with the previously obtained short reads for 79 isolates, encoding a total of 84 carbapenemase genes 
170 (Supplementary Table 3). The total number of contigs in the resulting hybrid assemblies ranged from 2-44 (median, 9). In 61/79 (77.2\%) hybrid assemblies, the largest contig was $\geq 5 \mathrm{Mb}$, indicating that all, or most, of the chromosomal sequence assembled into a single contig. The assemblies contained 1-8 plasmid replicons (median, 4), which are sequences used for defining plasmid incompatibility (Inc) groups (Carattoli et al. 2014). Multiple plasmid replicons were commonly found on the same contig, representing fusions between different plasmid types.

We found one copy of each carbapenemase gene in the hybrid assemblies.

178 Five (3x blaoxA-48-like, 1x blakPC, 1x blavim) were located on contigs ranging in size from 3.3-5.4Mb, each representing either a partial or putatively complete chromosomal sequence. The remaining 79 genes were located on contigs ranging in size from 2.5$313.6 \mathrm{~kb}$, which are hereafter described as putative plasmid sequences. Indeed, a plasmid origin is supported by the circularisation of $44(55.7 \%)$ of these sequences, as well as the identification of plasmid replicons in 65 (82.3\%). Of 11/79 (13.9\%) putative plasmid sequences that could neither be circularised nor contained plasmid replicons, we found additional evidence of a plasmid origin for ten (see Supplementary Note).

Dissemination of blaoxA-48-like genes by rapid spread of pOXA-48-like plasmids across diverse lineages

Amongst the 14 blaoxA-48-like-carrying hybrid assemblies obtained, we found the carbapenemase gene in three chromosomal sequences (3.3-5.4Mb) and 11 putative plasmid sequences (2.5-149.6kb) (Supplementary Table 3). The two isolates sequenced as GC group representatives harboured blaoxA-48-like on IncX3 and IncA/C2 plasmids, although we also found IncL/M(pOXA48) ( $n=3), \operatorname{IncL} / \mathrm{M}(\mathrm{pMU} 407)(n=1)$ and ColKP3 ( $n=3$ ) plasmids carrying blaoxA-48-like amongst the additional hybrid assemblies. Notably, three IncL/M(pOXA48) plasmids of $61.1-63.5 \mathrm{~kb}$ showed high structural and nucleotide similarity to a well-described, 61.8kb plasmid, pOXA-48a from strain 11978 (Poirel et al. 2012), which belongs to the pOXA-48-like family (Supplementary Figure

\section{1; see Supplementary Note).}

We determined the prevalence of the different blaoxA-48-like-carrying plasmid sequences across all blaoxA-48-like-carrying isolates in the sample collection $(n=249)$ by 
mapping the short sequence reads to each of the putative plasmid sequences obtained from the hybrid assemblies (see Methods). Importantly, this approach cannot reveal whether there have been insertions or rearrangements relative to the reference plasmid, or whether a particular resistance gene (in this case, blaoxA-48-like) is integrated into the same plasmid or located elsewhere, but nevertheless provides an indication of how much of each plasmid backbone is present.

Using this approach, we found that the IncX3, IncA/C2, IncL/M(pMU407) and ColKP3 plasmid sequences were found in full only rarely amongst all 249 isolates (Figure 1A; see Supplementary Note). In contrast, 204/249 (81.9\%) isolates had short reads that mapped to $\geq 99 \%$ of the circularised $63.5 \mathrm{~kb}$ IncL/M(pOXA48) plasmid from EuSCAPE_MT005 (and 221/249 (88.8\%) to $\geq 90 \%$ ). These comprised 202 isolates with the blaoxA-48 variant and two with blaoxA-162. For non-blaoxA-48-like-carrying isolates from the sample collection $(n=1468)$, the median length of mapping to this plasmid was just $2.9 \%$ (interquartile range, $2.4-4.9 \%$ ) while only $20 / 1468(1.4 \%)$ mapped to $\geq 90 \%$ of the plasmid length. Of these 20 , we found that six actually possessed blaoxA-48-like but at a lower coverage than the threshold previously used for determining presence/absence (David et al. 2019). These findings demonstrate a strong association between presence of blaoxA-48-like and the pOXA-48-like plasmid.

Isolates carrying blaoxA-48-like and possessing $\geq 99 \%$ of the IncL/M(pOXA48) plasmid sequence belonged to 37 STs across the K. pneumoniae species complex, and were submitted from 79 hospitals in 19 countries. These findings demonstrate the widespread nature of this plasmid. They also support a high frequency of carriage of blaoxA-48-like by the pOXA-48-like plasmid as they rule out the possibility of a spurious association caused by lineage or geographic effects.

Despite the broad distribution of pOXA-48-like plasmids amongst chromosomal backgrounds, $122 / 204(59.8 \%)$ of blaoxA-48-like-carrying isolates possessing $\geq 99 \%$ of this plasmid sequence belonged to one of three high-risk clonal lineages identified previously (ST11, ST15, ST101 and their derivatives) (David et al. 2019). This is approximately twice the value expected by chance (mean: 29.8\%, 95\% confidence intervals: $29.2-30.4 \%$ ) if the distribution of pOXA-48-like plasmids mirrored the relative abundance of these clonal lineages in the sample collection (see Methods).

We next performed phylogenetic analysis of pOXA-48-like plasmid sequences from 202 blaoxA-48-like-carrying isolates, which included those with both mapped sequence reads and bases called $(A / T / C / G$ rather than $N)$ at $\geq 90 \%$ of reference 
positions. In the absence of a known outgroup, the resulting phylogenetic tree was midpoint rooted (Figure 1B). One hundred and seventy-six (87.1\%) plasmid sequences were positioned on the ancestral node of a single main lineage or within two SNPs of this. Using published evolutionary rates for K. pneumoniae of $1.9 \times 10^{-6}$ SNPs/site/year and $3.65 \times 10^{-6} \mathrm{SNPs} /$ site/year (Mathers et al. 2015; Stoesser et al. 2014), we estimated that the time taken for a single SNP to occur across a $63.5 \mathrm{~kb}$ plasmid would range from 4.3 to 8.3 years. This assumed that evolutionary rates for chromosomes and plasmids are similar, which is likely given that they use the same cellular replication machinery, and are exposed to the same cellular environment. Since most pOXA-48-like sequences differ from a single ancestral variant by no more than two SNPs, this suggests that they have descended from a common ancestor that existed no more than 17 years prior to sampling (i.e. post 1996).

A tanglegram linking the plasmid-based and core genome-based phylogenies shows sharing of plasmid variants between different core genome lineages, providing clear evidence of plasmid horizontal transfer (Figure 1B). This has occurred frequently between core genome lineages that are co-localised at a country level. However, the core genome tree also contains 36 clonal expansions of isolates that each carry a particular plasmid variant, indicative of substantial vertical transmission. The largest contains 19 isolates from ST101, submitted from three hospitals across Romania.

Finally, all three hybrid assemblies harbouring the blaoxA-48 variant in the chromosome carried the gene within a Tn6237 composite transposon, which is a $\sim 20 \mathrm{~kb}$ sequence that also carries blaoxA-48-like in the pOXA-48-like plasmids

(Supplementary Figure 2). We found evidence of at least two independent chromosomal integrations of Tn6237 in ST11 and ST530, respectively, and subsequent clonal spread (see Supplementary Note).

Spread of blavim and blandm genes mediated by transient associations of diverse plasmids with multiple lineages

We obtained hybrid assemblies carrying blavIM genes representing the ten GC groups identified (Supplementary Table 3). Amongst these, we found blavIM in putative plasmid sequences (46.0-284.3kb) in 9/10 hybrid assemblies and in one 
blavIM was obtained from an isolate harbouring two carbapenemase genes (carrying also blakPC) but excluded from further analyses due to the short contig length $(2.9 \mathrm{~kb})$. We also obtained hybrid assemblies carrying blanDM genes representing the $15 \mathrm{GC}$ groups identified (Supplementary Table 3). All carried blanDM on putative plasmid

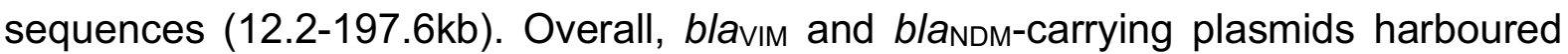
diverse replicon types. Several also shared partial structural and/or sequence homology.

The same short-read mapping approach used previously allowed us to determine the prevalence of the different plasmid sequences across all blaviM $(n=56)$

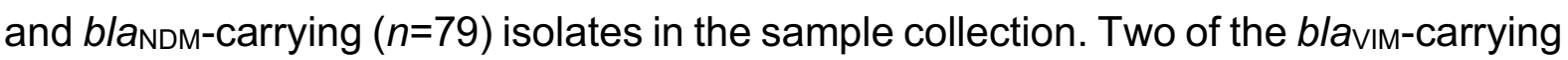
plasmids (from EuSCAPE_LV006 and EuSCAPE_IT312) and one of the blanDMcarrying plasmids (from EuSCAPE_RS064) were mapped over $\geq 90 \%$ by only the longread sequenced isolate and thus were unique in this collection (Figure 2). However, many plasmids were associated with clonal expansions of isolates, which were defined as two or more same-ST isolates clustered in the core genome-based phylogeny. In particular, 39/56 (69.6\%) blavıм-carrying isolates belonged to six clonal expansions and $38 / 79(48.1 \%)$ blanDM-carrying isolates belonged to seven clonal expansions, each with $\geq 99 \%$ mapping to a particular plasmid. Isolates from ST11, ST15 and ST101 (and their derivatives) accounted for the majority (56.4\% and $71.1 \%$, respectively) of these.

The core genome diversity within clonal expansions associated with particular plasmids was typically low with maximum pairwise SNP differences per clonal expansion ranging from 0-51 (median, 8) for blaviM-carrying isolates and 8-54 (median, 17) for blandM-carrying isolates. Given the published mutation rates (Mathers et al. 2015; Stoesser et al. 2014), we estimated the time taken for two isolates to diverge from a common ancestor by 54 SNPs would be 3.0 to 5.8 years. This is suggestive of recent acquisition of the plasmids by these lineages, and indicates that associations between the chromosome and these plasmids may be often only transient. Indeed, 4/6 and 2/7 clonal expansions of bla $\mathrm{VIM}_{\mathrm{M}}$-carrying and bla $\mathrm{NDM}_{\mathrm{N}}$-carrying isolates were restricted to a single hospital (and correspondingly have few SNP differences). A further $2 / 6$ and $1 / 7$, respectively, contained isolates submitted from different hospitals in the same country, while 4/7 of those with blanDM-carrying isolates were from different countries. While isolates from the three high-risk clonal lineages constituted $7 / 13$ of 
the total clonal expansions associated with particular blaviM and blanDM plasmids, they accounted for $6 / 7$ of those that had spread to multiple hospitals or countries.

We found some indications of plasmid sharing between STs, with three circularised blavim-carrying plasmids (from EuSCAPE_GR073, EuSCAPE_ES220 and EUSCAPE_RO094) and two circularised bla $a_{\mathrm{NDM}}$-carrying plasmids (from EuSCAPE_IE008 and EuSCAPE_RS010) mapped with short reads across $\geq 99 \%$ of their length by isolates from different STs (Figure 2). These often included isolates from different STs submitted from the same country but never the same hospital. Notably, they included a $68.4 \mathrm{~kb}$ blavim-1-carrying plasmid with high similarity to the pOXA-48-like plasmids that was recovered from the hybrid assembly of a ST483 isolate (EuSCAPE_ES220) but found also in ST11 and ST15 using short-read mapping (Figure 1B and Supplementary Figure 3; see Supplementary Note).

While plasmids shared between lineages were in general observed rarely, we found one exception, which was a non-circularised blanDM-carrying InCA/C2 plasmid sequence recovered from a ST274 isolate (EuSCAPE_RS105). This was mapped across $\geq 99 \%$ of its length by another eight ST274 isolates but also four ST101, two ST147 and one ST437 isolate(s). Long-read sequencing of one of the ST101 isolates (EuSCAPE_RS017) demonstrated that this IncA/C2 sequence formed part of a larger plasmid sequence in this isolate comprising both IncA/C2 and IncR replicons. We could not find the additional plasmid sequence, nor evidence of an IncR replicon, within the assembly of EuSCAPE_RS105. However, only three SNPs were found across $101.4 \mathrm{~kb}$ of shared sequence between the two plasmids (Supplementary Figure 4), suggestive of recent common ancestry.

\section{Dissemination of blaKPC genes by stable association with ST258/512 despite} frequent mobilisation between diverse plasmids

We obtained 44 bla $\mathrm{KPC}$-carrying hybrid assemblies from isolates representing 43 GC groups. These included two isolates from the same group selected to investigate possible plasmid transfer between STs (for details on these, see Supplementary Note; Supplementary Figure 5). The blakPC gene was found on a chromosomal sequence $(3.8 \mathrm{Mb})$ in one hybrid assembly, and on putative plasmid sequences (7.9-313.6kb) in the remaining 43. We found diverse replicon types 
amongst these putative plasmids, including those from the single clonal lineage of ST258/512. This lineage contains $230 / 312(73.7 \%)$ of all blakPC-carrying isolates in the sample collection.

Pairwise sequence comparisons between 24 circularised blakPC-carrying plasmids indicated that 15 were structural variants of two major IncF backbone types: backbone I ( $n=9)$ and backbone II $(n=6)$ (Figure 3). The first backbone type represents pKpQIL-like plasmids and we found that two have an identical size and structure to the originally described pKpQIL plasmid (Leavitt et al. 2010) (Supplementary Figure 6). The second backbone type shares sequence with pKPN3 (accession number, CP000648) but also pKpQIL-like (backbone I) plasmids (Supplementary Figure 7). Both backbone types I and II were found in ST258/512 and were geographically dispersed. Overall we found poor concordance between the plasmid types carrying bla KPC genes and the phylogeny of the host strain, including within ST258/512 (Figure 3).

Short read mapping of all blakpc-carrying isolates in the sample collection ( $n=312)$ to the newly-obtained blaKPC-carrying plasmids demonstrated that some (i.e. the IncP6, IncN and IncFIB(pKPHS1) plasmids) were found only rarely (Supplementary Figure 8). However, many isolates carry two or more of the reference plasmids, including 66 ST258/512 isolates that have $\geq 99 \%$ mapping to four distinct plasmid types with CoIRNAI, IncX3, IncFII(K)/IncFIB(pQIL) (i.e. backbone I) and IncFII $(K) / I n c F I B(K)$ (i.e. backbone II) replicons. This means that several of the reference plasmid sequences are frequently present in the same isolate, either in the same or a different structural arrangement, and we cannot infer which one (or more) contains the carbapenemase gene using the short sequence reads.

We therefore used an alternative approach that takes advantage of blakPC genes typically being on longer contigs in the short-read genome assemblies than other carbapenemases (Table 1). We compared each of the short-read contigs harbouring blaKPC genes $(n=312)$ with each of the 24 circularised blakPC-carrying plasmids from the hybrid assemblies. If $\geq 98 \%$ of the contig sequence could be aligned to a plasmid, we considered this as a match (Supplementary Figure 9; see Supplementary Note). We found that $28 / 82$ (34.1\%) of short-read contigs from nonST258/512 isolates matched either backbone I or II plasmids. This contrasted with $200 / 230(87.0 \%)$ of contigs from ST258/512 isolates. Of these $200,183(79.6 \%$ of 230) were not compatible with any other plasmid types. These results support 
backbones I and II (or related variants of these) being the dominant vectors of blakPC genes in ST258/512. However, only 36/230 (15.7\%) and 28/230 (12.2\%) ST258/512 contigs could be unambiguously assigned to either backbone I or II, respectively.

We next aimed to understand the evolutionary processes that have led to blakPC genes being carried on diverse plasmids within ST258/512, with a low degree of congruence between the plasmid type carrying blakPC and the core genome-based phylogeny. First, we determined if the plasmids on which we found blakPC are stably associated with the ST258/512 lineage or have been acquired repeatedly from outside of the lineage. We constructed phylogenetic trees of $91 \mathrm{pKpQIL-like} \mathrm{plasmids} \mathrm{and} 135$ IncX3 plasmids from ST258/512 isolates. These were from isolates that had short reads that mapped to $\geq 99 \%$ of the plasmid reference sequences, and comprised $48.9 \%$ and $95.1 \%$ of ST258/512 isolates possessing a IncFIB(pQIL) and IncX3 replicon, respectively. Comparisons of these plasmid-based trees with a core genomebased phylogeny of ST258/512 isolates revealed shared evolutionary histories, suggestive of single acquisitions early in the lineage history (Figure 4). IncX3 plasmid sequences with and without a blakPC gene (as confirmed using the hybrid assemblies) were also intermingled in the phylogenetic tree of IncX3 plasmids, further indicative of vertical propagation of the plasmid within the lineage coupled with occasional gain and/or loss of blaKPC (Figure 4B). While phylogenetic reconstructions were not undertaken for the CoIRNAI plasmid (due to a lack of diversity) or the backbone II plasmid (due to very high gene content variation), sequence comparisons of these plasmids with and without blaKPC genes showed high similarity between their backbones, indicative of a common origin (Supplementary Figures 10 and 11; see Supplementary Note). These findings suggest that blakPC genes can be maintained by plasmid backbones that are stable within the lineage. However, they do not reveal whether bla KPC genes have moved between plasmids within a single cell, or whether they have repeatedly been imported into ST258/512 plasmids from other strains (from either within or outside of ST258/512).

To distinguish between these possibilities, we next used the TETyper tool (Sheppard et al. 2018), which takes short-read data as input, to screen all blakPCcarrying isolates for the $\sim 10 \mathrm{~kb}$ Tn4401 transposon and investigate its patterns of inheritance. This transposon is known from previous studies to be the major carrier of blakPC genes in K. pneumoniae, especially amongst European strains (Cuzon et al. 2011; Chen et al. 2014). Tn4401 sequences were found in 229/230 (99.6\%) 
407 ST258/512 isolates harbouring blakPc genes, and classified into "combined" variants 408 based on both structural and SNP variation. We found two predominant combined 409 variants, Tn4401a-1 $(n=42)$ and Tn4401a-2 $(n=176)$, which differ by a single SNP that 410 also distinguishes the blaKPC-2 and blaKPC-3 gene variants. These variants correlate well 411 with the core genome-based phylogeny of ST258/512, with $42 / 46(91.3 \%)$ isolates in 412 one major clade (clade 1) carrying Tn4401a-1, and 175/184 (95.1\%) isolates in the 413 second major clade (clade 2) carrying Tn4401a-2 (Figure 5). This indicates a single 414 major acquisition of Tn4401 (carrying blakPC) by an ancestor of this lineage, followed 415 by relatively stable association during the clonal expansion of ST258/512. Taken 416 together, the combined stability of both the plasmids and Tn4401 transposon within 417 the ST258/512 lineage suggests that Tn4401 (carrying blakPC) has moved primarily 418 between plasmids in the same bacterial cell or between genetically identical cells 419 (such as those in a clonal infection). We also cannot rule out movement of Tn4401 420 between plasmids from different strains, provided that these strains are from the same 421 major clade of ST258/512 (i.e. possess the same combined variant of Tn4401). 422 However, the data is not compatible with frequent movement of Tn4401 between 423 strains from different major clades, or frequent import of Tn4401 into ST258/512 from outside of the lineage.

Finally, we investigated whether Tn4401 (and blakPC) has moved between plasmids via transposition or as part of larger recombination events. Using the shortread data with TETyper, we found identical 10bp flanking regions (CCAGCATTGA/ATTGAGTACC) upstream and downstream of Tn4401 in 176/230 (76.5\%) ST258/512 isolates, which include 5bp ATTGA target site duplications (Figure 5). Amongst blakPc-carrying plasmids from the hybrid assemblies, these particular flanking regions were restricted to backbone I and II plasmids (and three putative plasmid sequences with no known replicons). Taking these flanking regions to be markers of backbone I and II plasmids, these findings further support the dominant role of the two backbone types as vectors of blakPC genes. They also indicate that blakPC genes are typically mobilised between these plasmid types by larger recombination events (i.e. $>10 \mathrm{~kb}$ ) which transfer Tn4401 together with additional flanking sequence. Indeed we found a shared $34 \mathrm{~kb}$ sequence region around blakPC in plasmids representing backbones I and II that were recovered from closely-related isolates (EuSCAPE_GR049 and EuSCAPE_MK006) (Supplementary Figure 12).

440 Only two SNPs were found across this region, suggestive of recent transfer, in contrast 
441 to several hundred SNPs found across the remaining homologous sequence.

442 Conversely, we found distinct Tn4401 flanking regions in the CoIRNAI, IncX3, and 443 IncFIA(pKB30683) plasmids carrying blakPC genes in the hybrid assemblies, indicative 444 of transposition of Tn4401 into these plasmids (Figure 5). We also identified two 445 different flanking regions both upstream and downstream of Tn4401 in five ST258/512 446 isolates, suggesting that blakPC is present in two copies. However, none of these five 447 isolates were long-read sequenced to verify this.

\section{Discussion}

Molecular and genomic surveillance systems for bacterial pathogens currently rely on tracking clonally-evolving lineages. By contrast, extra-chromosomal plasmids, which can spread horizontally between strains and even species (Mathers et al. 2011; Martin et al. 2017), are usually excluded or analysed with low-resolution techniques (such as Inc typing). This is despite plasmids being the primary carriers of antibiotic resistance genes across many key pathogens. Here, we used combined long- and short- read sequencing of isolates from a European structured survey (EUSCAPE) (Grundmann et al. 2017; David et al. 2019) to investigate the diversity, distribution and transmission dynamics of resistance plasmids in K. pneumoniae. We focused on plasmids carrying carbapenemase genes, which confer resistance to carbapenems, a last-line class of antibiotics. We identified three major patterns by which carbapenemase genes have disseminated via plasmids, summarised as using one plasmid/multiple lineages (blaoxA-48-like), multiple plasmids/multiple lineages (blavim and blanDM) and multiple plasmids/one lineage (blaKPC). Despite these contrasts, our work revealed the high dependency of all three modes of carbapenemase gene spread on a small number of high-risk clones.

Previous studies have demonstrated a dominance of high-risk clones among antibiotic-resistant K. pneumoniae infections (Munoz-Price et al. 2013), although the reasons driving their success are still debated (Mathers et al. 2015). Here we have shown that carbapenemase-carrying plasmids are acquired by diverse lineages, such as the pOXA-48-like plasmid that was found in 37 STs. Yet our phylogenetic analyses 
indicate that carbapenemase-carrying plasmids are (i) non-randomly associated with high-risk clones (i.e. ST11, ST15, ST101, ST258/512), (ii) propagating by clonal expansion, and (iii) frequently spreading across healthcare networks and national borders. These findings reinforce the importance of preventing transmission, particularly of high-risk STs, through early detection and rigorous infection control.

The first blaoxA-48-carrying isolate described in 2004 (Poirel et al. 2004) was later found to carry the carbapenemase gene within a IncL/M pOXA-48-like plasmid (Poirel et al. 2012). Since then, numerous studies have reported this plasmid as the dominant vector of blaoxA-48-like genes both within and outside of Europe, and in both K. pneumoniae and other Enterobacterales species (Skalova et al. 2017; Potron et al. 2013). It has also since been further distinguished as an IncL plasmid, after IncL and IncM plasmids were found to be genetically distinct and compatible (Carattoli et al. 2015). Experiments performed by Adler et al. (2016) demonstrated that pOXA-48-like plasmids show very efficient conjugation both within and between bacterial species, helping to explain their predominance. Here, our comparative analyses demonstrate a single main acquisition of a blaoxA-48-like gene by a pOXA-48-like backbone. They suggest that almost all pOXA-48-like plasmids in our collection share a common ancestor that existed fewer than 17 years prior to the sample collection (i.e. post 1996). These plasmids have since spread rapidly as we found them in 79 hospitals in 19 countries across Europe. Most notably, we have shown that, despite frequent horizontal transfer of the plasmid, this onward spread has been primarily driven by the clonal expansion of high-risk STs.

By contrast, we found blavim and blandm genes on multiple diverse plasmids, which is concordant with reports from the literature (Samuelsen et al. 2011; PerezVazquez et al. 2019). As with the pOXA-48-like plasmid, our results show that clonal expansions, especially of high-risk STs, have driven the spread of these plasmids. We noted that associations of clonal lineages with plasmids carrying blavIM and blanDM genes were mostly recent, suggesting that they may be typically only transient. While the maximum age of any clonal expansion associated with a particular plasmid was estimated to be 5.8 years, most were much younger than this. We also found that plasmid sharing between lineages was coupled with structural changes in the plasmids that accumulate over time. We propose that high rates of recombination and rearrangement amongst plasmids could partially explain both the transient 
associations between lineages and plasmids, as well as the absence of any single dominant plasmid found across multiple lineages.

The most striking example of the reliance on high-risk clones is provided by the blakPC gene. Since its discovery in 1996, this gene has disseminated worldwide at a remarkable pace (Munoz-Price et al. 2013). A single clonal lineage, ST258/512, accounted for $>70 \%$ of all blakPC genes found amongst the EuSCAPE sample collection (David et al. 2019). Previous studies have shown that blaкPc can be carried by different plasmids in ST258/512 (Pitout et al. 2015; Noll et al. 2018). In particular, the pKpQIL-like (backbone I) plasmids have been highlighted as important vectors in North America, Europe and the Middle East (Chen et al. 2014; Papagiannitsis et al. 2016; Doumith et al. 2017). However, the origin of the different blakPc-carrying plasmids was unknown. Here we have shown that several of the key plasmid types carrying blakPC genes, including PKpQIL-like plasmids, are stably associated with the ST258/512 lineage. Our data support a single acquisition of blakPC by an early ancestor of the lineage, followed by movement of the gene between different plasmid types in the same bacterial cell. This was coupled with frequent recombination and rearrangement events between different plasmid types, leading to a complex array of mosaic structures carrying blakPC genes in the ST258/512 lineage.

We acknowledge several limitations of our study. First, we required carbapenemase-carrying contigs in the short-read assemblies to have $\geq 4$ genes to be used for defining GC groups, which then guided selection of isolates for long-read sequencing. This may have reduced the amount of plasmid diversity captured by disregarding isolates carrying carbapenemase genes within particular repetitive structures. Second, we used only one isolate from each GC group for long-read sequencing. This meant we were unable to assess the structural diversity and evolution of plasmids within shorter timescales, such as within clonal expansions of blavIM and blanDM-carrying isolates. We could also not confirm the stable presence of the carbapenemase gene on particular plasmids within these clonal expansions. Finally, the use of short-read mapping to reference plasmids had varying levels of utility and appropriateness. While it was useful for identification and phylogenetic analyses of stable plasmids (e.g. the pOXA-48-like plasmid), the common occurrence of mosaic plasmids could make these data difficult to interpret. 
In summary, we have highlighted three major modes of carbapenemase gene spread via plasmids. Consideration of each will be vital for incorporation of highresolution plasmid data into more comprehensive genomic surveillance systems.

\section{Materials \& Methods}

Clustering of short-read assembly contigs carrying carbapenemase genes into genetic context (GC) groups

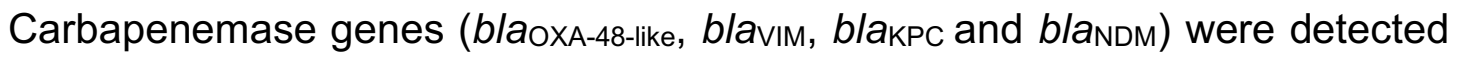
in the previously generated short-read assemblies (David et al. 2019) using BLASTn (Altschul et al. 1990). A minority of carbapenemase genes were not found in the assemblies despite detection using raw sequence reads. Conversely, carbapenemase genes that were detected in the assemblies but found with low coverage using the raw sequence reads ( $<0.2 x$ the coverage of the MLST gene with the lowest coverage) were excluded. All remaining contigs carrying carbapenemase genes were extracted from the short-read assemblies and annotated using Prokka v1.5 (Seemann, 2014). Contigs with four or more genes (including the carbapenemase) were used in the subsequent clustering analysis.

Annotated contigs containing a particular carbapenemase gene were used as input to Roary v3.11.3 (Page et al. 2015) to cluster the genes from different contigs into groups based on their nucleotide identity. Roary was run using default settings with the exception of the addition of the "-s" flag to prevent genes that are presumed to be paralogous being split into different gene groups. Contigs were excluded if the carbapenemase gene lacked a proper start or stop codon, as detected by Roary.

The remaining contigs were assigned to clustering groups based on the order and groupings of genes surrounding the carbapenemase (Table S1). Those with the same genes (according to the Roary gene groupings) in the same order around the carbapenemase were assigned to the same clustering group, while those with different genes and/or a different order were separated into different clustering groups. Contigs assigned to the same clustering group could be of different lengths and have different numbers of genes, with some contigs extending beyond others. Contigs could also 
belong to multiple clustering groups (i.e. if they matched multiple, usually larger contigs that are themselves different). Contigs that belonged to a single clustering group were assigned to a genetic context (GC) group, while those that belonged to multiple clustering groups were identified as having an "ambiguous" genetic context (Table S2). This clustering method is equivalent to finding all maximal cliques in a graph (Eppstein et al. 2013) and the solutions were obtained using a C++ script (https://github.com/darrenstrash/quick-cliques).

Culture, DNA extraction and long-read sequencing

Seventy-nine isolates were selected for long-read sequencing (Table S3). These include one isolate from each of the GC groups, with the exception of one blaoxA-48-like group and two blakPC groups for which representative isolates were unavailable. An additional eight blaoxA-48-like-carrying isolates were also included, while two blakpc-carrying isolates from the same GC group but different STs were selected to investigate possible within-hospital plasmid transfer.

The samples were grown on MacConkey agar plates at $37^{\circ} \mathrm{C}$ overnight and this was repeated until single colonies were visible on the plates. Single colonies of each sample were grown overnight in $7 \mathrm{~mL}$ of low salt lysogeny broth (LB) in a shaking incubator. Three $\mathrm{mL}$ of this culture was spun down at the maximum number of revolutions per minute (RPM) for three minutes and the supernatant was discarded. The pellet was resuspended in $500 \mu \mathrm{L}$ of lysis buffer, incubated at $80^{\circ} \mathrm{C}$ for five minutes and then cooled. Two hundred and seventy-five $\mu \mathrm{L}$ of $3 \mathrm{M}$ sodium acetate was added to the samples, which were vortexed briefly to mix. They were then spun at the maximum RPM for ten minutes. The supernatant was removed and added to clean Eppendorf tubes. Five $\mu \mathrm{L}$ of RNAse $A$ was added and the samples incubated for 30 minutes at $37^{\circ} \mathrm{C}$ before cooling. Two hundred $\mu \mathrm{L}$ of protein precipitate solution (Promega) was added, and the samples were incubated on ice for five minutes before being spun at the maximum RPM for three minutes. Finally, ethanol precipitation was performed.

Library preparation for all isolates was performed using the SMRTbell Template 
preparation. The Sequel data was demultiplexed using Lima in the SMRT link software (https://github.com/PacificBiosciences/barcoding).

607

Hybrid assembly

The long-read data were assembled together with the previously obtained short-reads for each sample (David et al. 2019) using the hybrid assembler, Unicycler v0.4.7 (Wick et al. 2017). Default settings were used for all samples with the exception

612 of four (EuSCAPE_IL075, EuSCAPE_DE024, EuSCAPE_ES089,

613 EuSCAPE_TR203). For these, we set the flag "--depth_filter", which represents the

614 fraction of the chromosomal depth below which contigs are filtered out, to 0.1 since

615 the carbapenemase genes were absent from the assemblies when the default setting

616 of 0.25 was used. Assembly statistics were generated using QUAST v.4.6.0 (Gurevich

617 et al. 2013). Assemblies were annotated using Prokka v1.5 (Seemann, 2014).

618 Annotated assemblies are available in the European Nucleotide Archive (ENA) and accession numbers can be found in Table S3.

620

Characterisation of hybrid assemblies

Contigs containing the carbapenemase genes were identified in the hybrid assemblies using BLASTn (Altschul et al. 1990). Replicon typing of all contigs was performed using Ariba v2.6.1 (Hunt et al. 2017) with the PlasmidFinder database (Carattoli et al. 2014). Galileo AMR (Partridge \& Tsafnat, 2018) was used to annotate mobile genetic elements within carbapenemase-carrying plasmids.

\section{Plasmid comparisons}

NUCmer v3.1 from the MUMmer package (Kurtz et al. 2004) was used to determine the length of sequence that could be aligned between pairs of plasmids, and the number of single nucleotide polymorphisms (SNPs) amongst the aligned regions. The Artemis Comparison Tool (ACT) v13.0.0 (Carver et al. 2005) was used to compare and visualise structural variation between two or more sequences.

637 to putative plasmid sequences obtained from the hybrid assemblies was used to 638 determine the length of the reference plasmid sequence present across isolates. 
Sequence reads were mapped using Burrows Wheeler Aligner (Li \& Durbin, 2009) and an in-house pipeline was used to identify SNPs using SAMtools mpileup v.0.1.19 and BCFtools v1.2 (Li et al. 2009). The length of the reference plasmid that was mapped by a minimum of one sequence read in each sample was determined from the BAM file. Upon testing of this approach, we found that the short reads of each carbapenemase-carrying isolate from which we obtained a hybrid assembly mapped to $99.8-100 \%$ (median, 100\%) of the putative carbapenemase-carrying plasmid sequence from the hybrid assembly.

Core genome-based phylogenetic analyses blandM-carrying isolates of $K$. pneumoniae sensu stricto were each constructed using variable positions within an alignment of 2539 genes. These represent loci that were found to be "core" genes (i.e. present in at least $95 \%$ of isolates within each species of the K. pneumoniae species complex) in previous analyses (David et al. 2019). Phylogenetic trees were inferred using RAxML v8.2.8 (Stamatakis, 2006) and midpoint-rooted. The same core genome alignment was also used to calculate pairwise SNP differences between isolates.

The phylogenetic tree of the ST258/512 lineage was constructed by mapping the short reads of the isolates to an ST258 reference genome (NJST258_1 (Deleo et al. 2014)). Recombined regions were removed from the pseudo-genome alignment using Gubbins v1.4.10 (Croucher et al. 2015) and the variable sites in the resulting alignment were used as input to RAxML v8.2.8 (Stamatakis, 2006). The tree was rooted based on previous phylogenetic analyses of the full sample collection that included outgroups of ST258/512 (David et al. 2019).

\section{Plasmid-based phylogenetic analyses}

To construct a phylogenetic tree of pOXA-48-like plasmids, we first generated a plasmid alignment comprising blaoxA-48-like-carrying isolates with bases mapped and called at $\geq 90 \%$ of the reference plasmid from EuSCAPE_MT005. This included plasmids from 203 isolates, although one plasmid sequence from the $K$. quasipneumoniae species was subsequently excluded. An additional five blaviмcarrying isolates with bases mapped and called at $\geq 90 \%$ of the reference plasmid were also included in the alignment. 

lineage were also constructed using the alignments generated from mapping of the short reads to reference plasmids. The references used were the pKpQIL-like plasmid of EuSCAPE_IT030 and the IncX3 plasmid of EuSCAPE_IL063. Isolates with $\geq 99 \%$ of bases called at the reference positions were included.

Variable positions in these plasmid alignments, excluding any positions containing an $N$ (rather than $A / T / C / G$ ) in $\geq 1$ isolate, were used to infer phylogenetic trees using RAxML v8.2.8 (Stamatakis, 2006). Nucleotide variants of plasmids were determined using the same alignments. Tanglegrams linking the core genome and plasmid phylogenies were generated using the "cophylo" function from the "phytools" package in R (https://www.r-project.org/).

Comparison of the actual and expected proportion of pOXA-48-like plasmids in highrisk lineages

We compared the actual proportion of pOXA-48-like plasmids carried amongst three high-risk clonal lineages (ST11, ST15, ST101) with the expected proportion if the distribution of plasmids reflected the relative abundance of these lineages in the population. Isolates belonging to other STs that have evolved from these three STs were included with them, with the exception of ST258/512. We first determined whether each isolate in the full EuSCAPE sample collection carried a pOXA-48-like plasmid, based on whether the short reads mapped to at least $\geq 99 \%$ of the reference plasmid obtained from the hybrid assembly of EuSCAPE_MT005. The actual proportion of isolates carrying a pOXA-48-like plasmid that belonged to one of the three high-risk lineages was calculated. The pOXA-48-like plasmids were then randomly re-distributed across all isolates in the sample collection and the proportion of pOXA-48-like plasmids in the three high-risk clonal lineages was re-calculated. This was repeated one hundred times, and the mean and $95 \%$ confidence intervals were obtained from these values.

Replicon typing of all short-read data al. 2017) with the PlasmidFinder database (Carattoli et al. 2014). 
706 Comparison of blakpc-carrying short-read contigs with complete plasmids with

707 NUCmer

708 Each short-read contig carrying a blakPC gene was compared with each of the

709 complete blakPC-carrying plasmids obtained from the hybrid assemblies using

710 NUCmer v3.1 (Kurtz et al. 2004). Contigs that could be aligned over $\geq 98 \%$ of their

711 length to a complete plasmid were deemed to match that plasmid.

712

Characterisation of Tn4401 variation

The variation within Tn4401 and its flanking regions was characterised using

715 TETyper v1.1 (Sheppard et al. 2018) taking the short reads of all blakPC-carrying 716 isolates as input.

\section{Data availability}

All raw long-read sequence data and hybrid assemblies are available from the

721 European Nucleotide Archive (ENA) under the study accession number, PRJEB33308

722 (ERP116089). Individual accession numbers for raw sequence data and hybrid assemblies are also available in Supplementary Table 3.

724

725

726

727

728

729

730 


\section{Tables}

Table 1. Number of isolates assigned to different genetic context (GC) groups of the

\begin{tabular}{|l|l|l|l|l|l|l|}
\hline $\begin{array}{l}\text { Carbapenemase } \\
\text { gene }\end{array}$ & $\begin{array}{l}\text { No. of } \\
\text { isolates }\end{array}$ & $\begin{array}{l}\text { Median } \\
\text { no. of } \\
\text { genes per } \\
\text { short-read } \\
\text { contig } \\
\text { (IQR) }\end{array}$ & $\begin{array}{l}\text { No. (\%) of } \\
\text { isolates } \\
\text { with } \\
\text { assigned } \\
\text { GC group }\end{array}$ & $\begin{array}{l}\text { No. (\%) of } \\
\text { isolates } \\
\text { discarded } \\
\text { from } \\
\text { clustering* }\end{array}$ & $\begin{array}{l}\text { No. (\%) of } \\
\text { isolates with } \\
\text { ambiguous } \\
\text { context }\end{array}$ & $\begin{array}{l}\text { No. of } \\
\text { GC } \\
\text { groups }\end{array}$ \\
\hline blaoxA-48-like & 249 & $2(2-2)$ & $4(1.6 \%)$ & $221(88.8 \%)$ & $24(9.6 \%)$ & 3 \\
\hline blavIM & 56 & $6(2-7)$ & $39(69.6 \%)$ & $16(28.6 \%)$ & $1(1.8 \%)$ & 10 \\
\hline blanDM & 79 & $15(4-22)$ & $59(74.7 \%)$ & $6(7.6 \%)$ & $14(17.7 \%)$ & 15 \\
\hline blakPC & 312 & $23(18-35)$ & $82(26.3 \%)$ & $0(0 \%)$ & $230(73.7 \%)$ & 45 \\
\hline
\end{tabular}

*Isolates were discarded from the clustering either because there were fewer than four genes on the carbapenemase-carrying contig, or if the carbapenemase gene was found in the assembly without a start or stop codon.

**Isolates were designated an "ambiguous" context if the carbapenemase-carrying contig matched multiple, different (often larger) contigs.

\section{Corresponding Author}

751 Correspondence to Sophia David (sophia.david@sanger.ac.uk) and David

\section{Aanensen (david.aanensen@bdi.ox.ac.uk)}




\section{Acknowledgements}

The authors would like to thank the Pathogen Informatics team and the DNA Pipelines Long read team at the Wellcome Sanger Institute for their contribution to the study.

\section{Author Contributions}

SD and DMA conceived the study. The EuSCAPE working group collected the bacterial isolates and epidemiological data. The ESGEM facilitated the training and capacity building for the collection of bacterial isolates. SD, VC, SR, AS, TG, JP, GMR, EJF, HG and DMA performed the data analysis. SD, GMR, EJF, HG and DMA wrote the manuscript. All authors read and approved the manuscript.

\section{Source of Funding}

770 This work was funded by The Centre for Genomic Pathogen Surveillance, Wellcome Genome Campus, Wellcome (grants 098051 and 099202) and The NIHR Global Health Research Unit on Genomic Surveillance of Antimicrobial Resistance (NIHR 16/136/111). The EuSCAPE project was funded by ECDC through a specific framework contract (ECDC/2012/055) following an open call for tender (OJ/25/04/2012-PROC/2012/036).

\section{Competing Interests}

779 The authors declare no competing interests. 


\section{The EuSCAPE working group}

Andi Koraqi ${ }^{7}$, Denada Lacej ${ }^{7}$, Petra Apfalter ${ }^{8}$, Rainer Hartl ${ }^{8}$, Youri Glupczynski ${ }^{9}$, Te-Din Huang ${ }^{9}$, Tanya Strateva ${ }^{10}$, Yuliya Marteva-Proevska ${ }^{11}$, Arjana Tambic Andrasevic 12, Iva Butic 12, Despo Pieridou-Bagatzouni 13, Panagiota MaikantiCharalampous ${ }^{13}$, Jaroslav Hrabak ${ }^{14}$, Helena Zemlickova ${ }^{15}$, Anette Hammerum ${ }^{16}$, Lotte Jakobsen ${ }^{16}$, Marina Ivanova ${ }^{17}$, Anastasia Pavelkovich ${ }^{17}$, Jari Jalava ${ }^{18}$, Monica Österblad ${ }^{18}$, Laurent Dortet ${ }^{19}$, Sophie Vaux ${ }^{20}$, Martin Kaase ${ }^{21}$, Sören G. Gatermann ${ }^{22}$, Alkiviadis Vatopoulos ${ }^{23}$, Kyriaki Tryfinopoulou ${ }^{23}$, Ákos Tóth ${ }^{24}$, Laura Jánvári ${ }^{24}$, Teck Wee Boo 25, Elaine McGrath 25, Yehuda Carmeli ${ }^{26}$, Amos Adler ${ }^{26}$, Annalisa Pantosti ${ }^{27}$, Monica Monaco ${ }^{27}$, Lul Raka ${ }^{28}$, Arsim Kurti ${ }^{28}$, Arta Balode ${ }^{29}$, Mara Saule 29 , Jolanta Miciuleviciene ${ }^{30}$, Aiste Mierauskaite ${ }^{30}$, Monique Perrin-Weniger ${ }^{31}$, Paul Reichert ${ }^{31}$, Nina Nestorova ${ }^{32}$, Sonia Debattista ${ }^{32}$, Gordana Mijovic ${ }^{33}$, Milena Lopicic ${ }^{33}$, Ørjan Samuelsen ${ }^{34}$, Bjørg Haldorsen ${ }^{34}$, Dorota Żabicka ${ }^{35}$, Elżbieta Literacka ${ }^{36}$, Manuela Caniça ${ }^{37}$, Vera Manageiro ${ }^{37}$, Ana Kaftandzieva ${ }^{38}$, Elena Trajkovska-Dokic ${ }^{38}$, Maria Damian ${ }^{39}$, Brandusa Lixandru ${ }^{39}$, Zora Jelesic ${ }^{40}$, Anika Trudic ${ }^{41}$, Milan Niks Pérez-Vázquez ${ }^{45}$, Christian Giske ${ }^{46}$, Karin Sjöström ${ }^{47}$, Deniz Gür ${ }^{48}$, Aslı Cakar ${ }^{48}$, Neil Woodford ${ }^{49}$, Katie Hopkins ${ }^{49}$, Camilla Wiuff ${ }^{50}$, Derek J. Brown ${ }^{51}$.

7 University Hospital Center "Mother Theresa", Tirana, Albania. 8 Elisabethinen Hospital Linz, Linz, Austria. ${ }^{9} \mathrm{CHU}$ Dinant-Godinne UCL Namur, Namur, Belgium. ${ }^{10}$

805 Faculty of Medicine, Medical University of Sofia, Sofia, Bulgaria. ${ }^{11}$ Alexandrovska 806 University Hospital, Sofia, Bulgaria. ${ }^{12}$ University Hospital for Infectious Diseases, 807 Zagreb, Croatia. ${ }^{13}$ Nicosia General Hospital, Nicosia, Cyprus. ${ }^{14}$ Faculty of Medicine 808 in Plzen, Charles University in Prague, Plzen, Czech Republic. ${ }^{15}$ National Institute of 809 Public Health, Praha, Czech Republic. 16 Statens Serum Institut, Copenhagen, 810 Denmark. ${ }^{17}$ East Tallinn Central Hospital, Tallinn, Estonia. ${ }^{18}$ National Institute for 811 Health and Welfare, Turku, Finland. ${ }^{19}$ Bicêtre Hospital, Le Kremlin-Bicêtre, France. ${ }^{20}$ 812 Institut de Veille Sanitaire, Saint-Maurice, France. ${ }^{21}$ Universitätsmedizin Göttingen,

813 Göttingen, Germany. ${ }^{22}$ Ruhr-University Bochum, Bochum, Germany. ${ }^{23}$ National 814 School of Public Health, Athens, Greece. ${ }^{24}$ National Center for Epidemiology, 815 Budapest, Hungary. ${ }^{25}$ Galway University Hospitals, Galway, Ireland. ${ }^{26}$ Ministry of 
816 Health, Tel-Aviv, Israel. ${ }^{27}$ Istituto Superiore di Sanità, Rome, Italy. ${ }^{28}$ National Institute

817 of Public Health of Kosovo, Prishtina, Kosovo. ${ }^{29}$ Pauls Stradins Clinical University

818 Hospital, Riga, Latvia. ${ }^{30}$ National Public Health Surveillance Laboratory, Vilnius,

819 Lithuania. ${ }^{31}$ Laboratoire National De Sante, Düdelingen, Luxembourg. ${ }^{32}$ Mater Dei

820 Hospital, Msida, Malta. ${ }^{33}$ Institute of Public Health, Podgorica, Montenegro. ${ }^{34}$

821 University Hospital of North Norway, Troms $\varnothing$, Norway. ${ }^{35}$ Narodowy Instytut Lekow,

822 Warsaw, Poland. ${ }^{36}$ National Medicines Institute, Warsaw, Poland. ${ }^{37}$ National Institute

823 of Health Dr. Ricardo Jorge, Lisbon, Portugal. 38 Institute of Microbiology and

824 Parasitology, Medical Faculty, Skopje, Republic of Macedonia. ${ }^{39}$ Cantacuzino

825 National Research Institute, Bucharest, Romania. ${ }^{40}$ Institute for Public Health of

826 Vojvodina, Novi Sad, Serbia. 41 Institute for Pulmonary Diseases of Vojvodina,

827 Sremska Kamenica, Serbia. ${ }^{42}$ Public Health Authority of the Slovak Republic,

828 Bratislava, Slovakia. ${ }^{43}$ University Hospital of P.J.Safarik, Kosice, Slovakia. ${ }^{44}$ Institute

829 of Microbiology and Immunology, Ljubljana, Slovenia. 45 Centro Nacional de

830 Microbiología, Instituto de Salud Carlos III, Madrid, Spain. ${ }^{46}$ Karolinska Institutet,

831 Stockholm, Sweden. ${ }^{47}$ Public Health Agency of Sweden, Stockholm, Sweden. ${ }^{48}$

832 Hacettepe University, Ankara, Turkey. ${ }^{49}$ National Infection Service, Public Health

833 England, London, United Kingdom - England and Northern Ireland. ${ }^{50}$ Sydvestjysk

834 Hospital, Esbjerg, Denmark. 51 Scottish Microbiology Reference Laboratories,

835 Glasgow, United Kingdom - Scotland.

836

837

838

839

840 


\section{References}

1. World Health Organization Global Priority List of Antibiotic-Resistant Bacteria to Guide Research, Discovery, and Development of New Antibiotics. World Health Organisation (2017).

2. Cassini A, Högberg LD, Plachouras D, Quattrocchi A, Hoxha A, Simonsen GS, et al. Attributable deaths and disability-adjusted life-years caused by infections with antibiotic-resistant bacteria in the EU and the European Economic Area in 2015: a population-level modelling analysis. Lancet Infect Dis. 2019;19(1):56-66.

3. David S, Reuter S, Harris SR, Glasner C, Feltwell T, Argimon S, et al. Epidemic of carbapenem-resistant Klebsiella pneumoniae in Europe is driven by nosocomial spread. Nat Microbiol. 2019;4(11):1919-29.

4. Mathers AJ, Cox HL, Kitchel B, Bonatti H, Brassinga AKC, Carroll J, et al. Molecular dissection of an outbreak of carbapenem-resistant Enterobacteriaceae reveals intergenus KPC carbapenemase transmission through a promiscuous plasmid. MBio. 2011;2(6).

5. Martin J, Phan HTT, Findlay J, Stoesser N, Pankhurst L, Navickaite I, et al. Covert dissemination of carbapenemase-producing Klebsiella pneumoniae (KPC) in a successfully controlled outbreak: long- and short-read wholegenome sequencing demonstrate multiple genetic modes of transmission. Journal of Antimicrobial Chemotherapy. 2017;72(11):3025-34.

6. Sheppard AE, Stoesser N, Wilson DJ, Sebra R, Kasarskis A, Anson LW, et al. Nested Russian doll-like genetic mobility drives rapid dissemination of the carbapenem resistance gene blaKPC. Antimicrob Agents Chemother. 2016;60(6):3767-78.

7. Aanensen DM, Feil EJ, Holden MTG, Dordel J, Yeats CA, Fedosejev A, et al. Whole-genome sequencing for routine pathogen surveillance in public health: a population snapshot of invasive Staphylococcus aureus in Europe. MBio. 2016;7(3).

8. Domman D, Quilici ML, Dorman MJ, Njamkepo E, Mutreja A, Mather AE, et al. Integrated view of Vibrio cholerae in the Americas. Science. 2017;358(6364):789-93. 
9. Harris SR, Cole MJ, Spiteri G, Sánchez-Busó L, Golparian D, Jacobsson S, et al. Public health surveillance of multidrug-resistant clones of Neisseria gonorrhoeae in Europe: a genomic survey. Lancet Infect Dis. 2018;18(7):75868.

10. Wick RR, Judd LM, Gorrie CL, Holt KE. Unicycler: Resolving bacterial genome assemblies from short and long sequencing reads. PLoS Comput Biol. 2017;13(6):e1005595.

11. George S, Pankhurst L, Hubbard A, Votintseva A, Stoesser N, Sheppard AE, et al. Resolving plasmid structures in Enterobacteriaceae using the MinION nanopore sequencer: assessment of MinION and MinION/Illumina hybrid data assembly approaches. Microb Genom. 2017;3(8):e000118.

12. Grundmann H, Glasner C, Albiger B, Aanensen DM, Tomlinson CT, Andrasevic AT, et al. Occurrence of carbapenemase-producing Klebsiella pneumoniae and Escherichia coli in the European survey of carbapenemaseproducing Enterobacteriaceae (EuSCAPE): a prospective, multinational study. Lancet Infect Dis. 2017;17(2):153-63.

13. Carattoli A, Zankari E, García-Fernández A, Voldby Larsen M, Lund O, Villa L, et al. In silico detection and typing of plasmids using PlasmidFinder and plasmid multilocus sequence typing. Antimicrob Agents Chemother. 2014;58(7):3895-903.

14. Poirel L, Bonnin RA, Nordmann P. Genetic features of the widespread plasmid coding for the carbapenemase OXA-48. Antimicrob Agents Chemother. 2012;56(1):559-62.

15. Mathers AJ, Stoesser N, Sheppard AE, Pankhurst L, Giess A, Yeh AJ, et al. Klebsiella pneumoniae carbapenemase (KPC)-producing K. pneumoniae at a single institution: insights into endemicity from whole-genome sequencing. Antimicrob Agents Chemother. 2015;59(3):1656-63.

16. Stoesser N, Giess A, Batty EM, Sheppard AE, Walker AS, Wilson DJ, et al. Genome sequencing of an extended series of NDM-producing Klebsiella pneumoniae isolates from neonatal infections in a Nepali hospital characterizes the extent of community- versus hospital-associated transmission in an endemic setting. Antimicrob Agents Chemother. 2014;58(12):7347-57. 
17. Leavitt A, Chmelnitsky I, Carmeli Y, Navon-Venezia S. Complete nucleotide sequence of KPC-3-encoding plasmid pKpQIL in the epidemic Klebsiella pneumoniae sequence type 258. Antimicrob Agents Chemother. 2010;54(10):4493-6.

18. Sheppard AE, Stoesser N, German-Mesner I, Vegesana K, Sarah Walker A, Crook DW, et al. TETyper: a bioinformatic pipeline for classifying variation and genetic contexts of transposable elements from short-read whole-genome sequencing data. Microb Genom. 2018;4(12).

19. Cuzon G, Naas T, Nordmann P. Functional characterization of Tn4401, a Tn3-based transposon involved in blaKPC gene mobilization. Antimicrob Agents Chemother. 2011;55(11):5370-3.

20. Chen L, Mathema B, Chavda KD, DeLeo FR, Bonomo RA, Kreiswirth BN. Carbapenemase-producing Klebsiella pneumoniae: molecular and genetic decoding. Trends Microbiol. 2014;22(12):686-96.

21. Munoz-Price LS, Poirel L, Bonomo RA, Schwaber MJ, Daikos GL, Cormican $\mathrm{M}$, et al. Clinical epidemiology of the global expansion of Klebsiella pneumoniae carbapenemases. Lancet Infect Dis. 2013;13(9):785-96. and international high-risk clones in the spread of multidrug-resistant Enterobacteriaceae. Clinical Microbiology Reviews. 2015;28(3):565-91. mediated resistance to imipenem in Klebsiella pneumoniae. Antimicrobial Agents and Chemotherapy. 2004;48(1):15-22.

24. Skalova A, Chudejova K, Rotova V, Medvecky M, Studentova V, Chudackova $\mathrm{E}$, et al. Molecular characterization of OXA-48-like-producing Enterobacteriaceae in the Czech Republic and evidence for horizontal transfer of pOXA-48-like plasmids. Antimicrob Agents Chemother. 2017;61(2). 25. Potron A, Poirel L, Rondinaud E, Nordmann P. Intercontinental spread of OXA-48 beta-lactamase-producing Enterobacteriaceae over a 11-year period, 2001 to 2011. Euro Surveill. 2013;18(31).

26. Carattoli A, Seiffert SN, Schwendener S, Perreten V, Endimiani A.

Differentiation of IncL and IncM Plasmids Associated with the Spread of Clinically Relevant Antimicrobial Resistance. PLoS One. 2015;10(5):e0123063. 
27. Adler A, Khabra E, Paikin S, Carmeli Y. Dissemination of the blaKPC gene by clonal spread and horizontal gene transfer: comparative study of incidence and molecular mechanisms. J Antimicrob Chemother. 2016;71(8):2143-6. TR, et al. Molecular characterization of VIM-producing Klebsiella pneumoniae from Scandinavia reveals genetic relatedness with international clonal complexes encoding transferable multidrug resistance. Clin Microbiol Infect. 2011;17(12):1811-6.

29. Pérez-Vázquez M, Sola Campoy PJ, Ortega A, Bautista V, Monzón S, RuizCarrascoso G, et al. Emergence of NDM-producing Klebsiella pneumoniae and Escherichia coli in Spain: phylogeny, resistome, virulence and plasmids encoding blaNDM-like genes as determined by WGS. J Antimicrob Chemother. 2019;74(12):3489-96.

30. Pitout JD, Nordmann P, Poirel L. Carbapenemase-producing Klebsiella pneumoniae, a key pathogen set for global nosocomial dominance. Antimicrob Agents Chemother. 2015;59(10):5873-84. diversity of carbapenemase-producing gram-negative bacteria using single molecule sequencing. bioRixv. 2018.

32. Chen L, Chavda KD, Melano RG, Jacobs MR, Koll B, Hong T, et al. Comparative genomic analysis of KPC-encoding pKpQIL-like plasmids and their distribution in New Jersey and New York Hospitals. Antimicrob Agents Chemother. 2014;58(5):2871-7.

33. Papagiannitsis CC, Di Pilato V, Giani T, Giakkoupi P, Riccobono E, Landini G, et al. Characterization of KPC-encoding plasmids from two endemic settings, Greece and Italy. J Antimicrob Chemother. 2016;71(10):2824-30.

34. Doumith M, Findlay J, Hirani H, Hopkins KL, Livermore DM, Dodgson A, et al. Major role of pKpQIL-like plasmids in the early dissemination of KPC-type carbapenemases in the UK. J Antimicrob Chemother. 2017;72(8):2241-8. search tool. J Mol Biol. 1990;215(3):403-10. 2014;30(14):2068-9. 
37. Page AJ, Cummins CA, Hunt M, Wong VK, Reuter S, Holden MT, et al. Roary: rapid large-scale prokaryote pan genome analysis. Bioinformatics. 2015;31(22):3691-3.

38. Eppstein D, Loffler M, Strash D. Listing all maximal cliques in large sparse real-world graphs in near-optimal time. Journal of Experimental Algorithmics. 2013;18(3).

39. Gurevich A, Saveliev V, Vyahhi N, Tesler G. QUAST: quality assessment tool for genome assemblies. Bioinformatics. 2013;29(8):1072-5.

40. Hunt M, Mather AE, Sanchez-Buso L, Page AJ, Parkhill J, Keane JA, et al. ARIBA: rapid antimicrobial resistance genotyping directly from sequencing reads. Microbial Genomics. 2017;3(10).

41. Partridge SR, Tsafnat G. Automated annotation of mobile antibiotic resistance in Gram-negative bacteria: the Multiple Antibiotic Resistance Annotator (MARA) and database. J Antimicrob Chemother. 2018;73(4):883-90.

42. Kurtz S, Phillippy A, Delcher AL, Smoot M, Shumway M, Antonescu C, et al. Versatile and open software for comparing large genomes. Genome Biology. 2004;5(2).

43. Carver TJ, Rutherford KM, Berriman M, Rajandream MA, Barrell BG, Parkhill J. ACT: the Artemis comparison tool. Bioinformatics. 2005;21(16):3422-3.

44. Li H, Durbin R. Fast and accurate short read alignment with Burrows-Wheeler transform. Bioinformatics. 2009;25(14):1754-60.

45. Li H, Handsaker B, Wysoker A, Fennell T, Ruan J, Homer N, et al. The Sequence Alignment/Map format and SAMtools. Bioinformatics. 2009;25(16):2078-9.

46. Stamatakis A. RAxML-VI-HPC: maximum likelihood-based phylogenetic analyses with thousands of taxa and mixed models. Bioinformatics. 2006;22(21):2688-90.

47. Deleo FR, Chen L, Porcella SF, Martens CA, Kobayashi SD, Porter AR, et al. Molecular dissection of the evolution of carbapenem-resistant multilocus sequence type 258 Klebsiella pneumoniae. Proc Natl Acad Sci. 2014;111(13):4988-93.

48. Croucher NJ, Page AJ, Connor TR, Delaney AJ, Keane JA, Bentley SD, et al. Rapid phylogenetic analysis of large samples of recombinant bacterial whole genome sequences using Gubbins. Nucleic Acids Res. 2015;43(3):e15. 


\section{Figures}

A
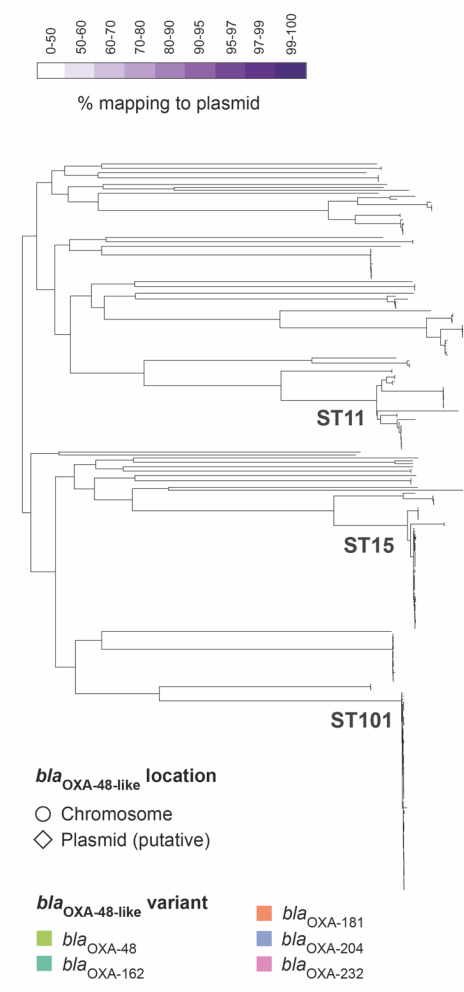

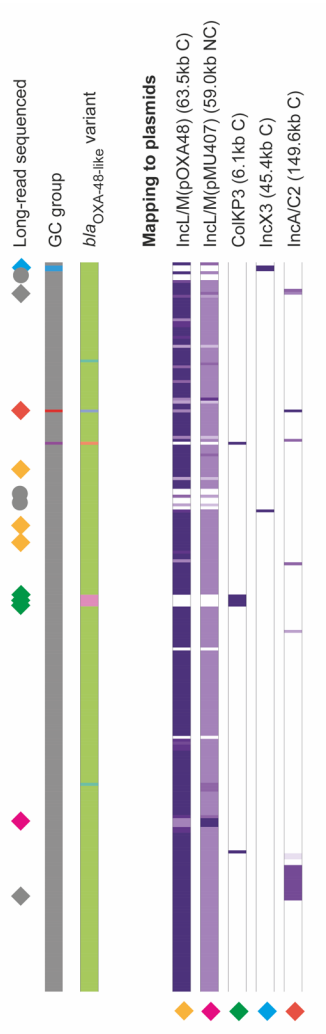

B

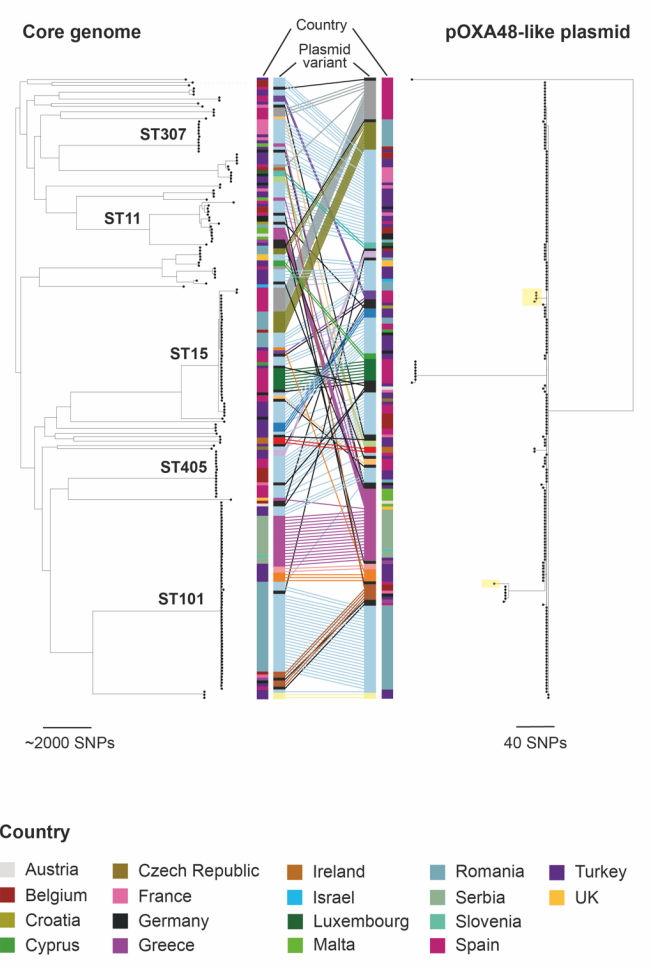

Figure 1. High prevalence of the pOXA-48-like plasmid sequence across blaoxA-48-likecarrying isolates. A) The phylogenetic tree includes 248 blaoxA-48-like-carrying isolates from $K$. pneumoniae sensu stricto (the single blaoxA-48-ike-carrying isolate from $K$. quasipneumoniae was excluded). It was constructed using SNPs in the core genome and midpoint-rooted. All non-blaoxA-48-like-carrying isolates, which would be interspersed amongst the isolates here, were also excluded. Long-read sequenced isolates are marked next to the tree with a diamond if they carry blaoxA-48-like on a putative plasmid sequence or a circle if they carry the gene on the chromosome. The colours of the diamonds represent distinct blaoxA-48-ike-carrying plasmids that were obtained. The first two columns, from left to right, show the genetic context (GC) group of isolates assigned using the short-read assembly contigs (ambiguous isolates not assigned to any group are in grey) and the blaoxA-48-like variant. Remaining columns show the percentage length of bla oxA-48-like-carrying plasmid sequences obtained from the hybrid assemblies that are mapped by short reads of the 248 blaoxA-48-ike-carrying isolates (note the non-linear colour gradient). Mapping is shown to single representatives of the IncL/M(pOXA48) (i.e. pOXA-48-like) and ColKP3 plasmids since several highly similar plasmids were obtained with each of these replicons. The five reference plasmids used are from isolates, EuSCAPE_MT005, EuSCAPE_TR057, EuSCAPE_TR009, EuSCAPE_BE078, and EuSCAPE_FR056 (left to right in figure). Each plasmid sequence is indicated by a diamond of the same colour as that indicating the isolate(s) in the tree from which the plasmid was recovered. Mapping data for two shorter blaoxA-48-like-carrying putative plasmids is not shown (EuSCAPE_RS017 - 20.3kb; EuSCAPE_TR203 - 2.5kb). C - circular; NC - noncircular. B) The tanglegram links phylogenetic trees constructed using SNPs in the core genome (left) and the pOXA48-like plasmid (right). Both trees are midpoint-rooted. The trees 
include 207 isolates from K. pneumoniae sensu stricto that had mapping and bases called (A/T/C/G rather than $N$ ) at $\geq 90 \%$ of positions in the plasmid reference sequence. These comprise 202 isolates with blaoxA-48-like genes and five with blavim genes, the latter of which are shaded in yellow in the plasmid tree. The blaoxA-48-like-carrying isolate from $K$. quasipneumoniae that possessed this plasmid is excluded. Lines are drawn between tips in the trees representing the same isolate and coloured by the nucleotide sequence variant of the plasmid. Unique plasmid variants are coloured black. The country of origin of each isolate is shown.

A

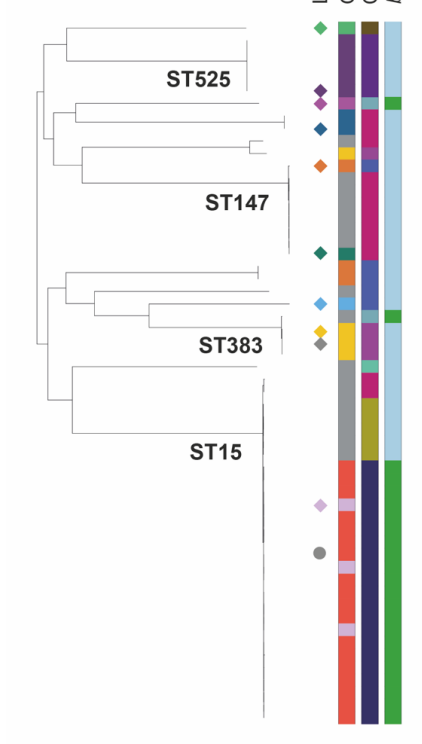

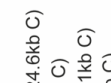
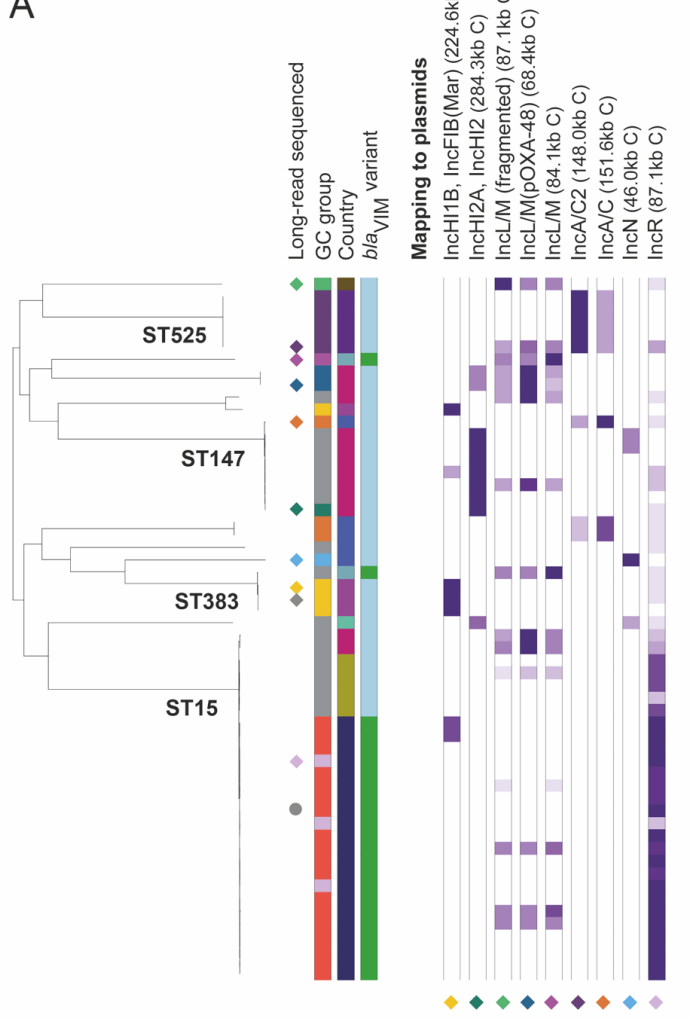

B

$b / a_{\mathrm{VIM}}$ and $b / a_{\mathrm{NDM}}$ location

C Chromosome

$\diamond$ Plasmid (putative)

bla $_{\mathrm{VIM}}$ variant

bla ${ }_{\mathrm{VIM}-1}$

- $b / a_{\mathrm{VIM}-4}$
Country

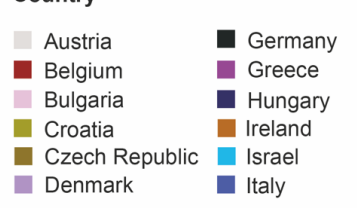

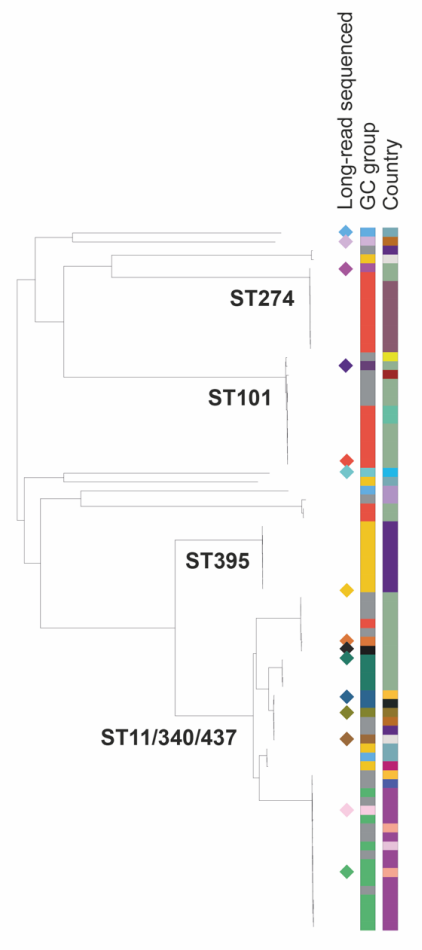
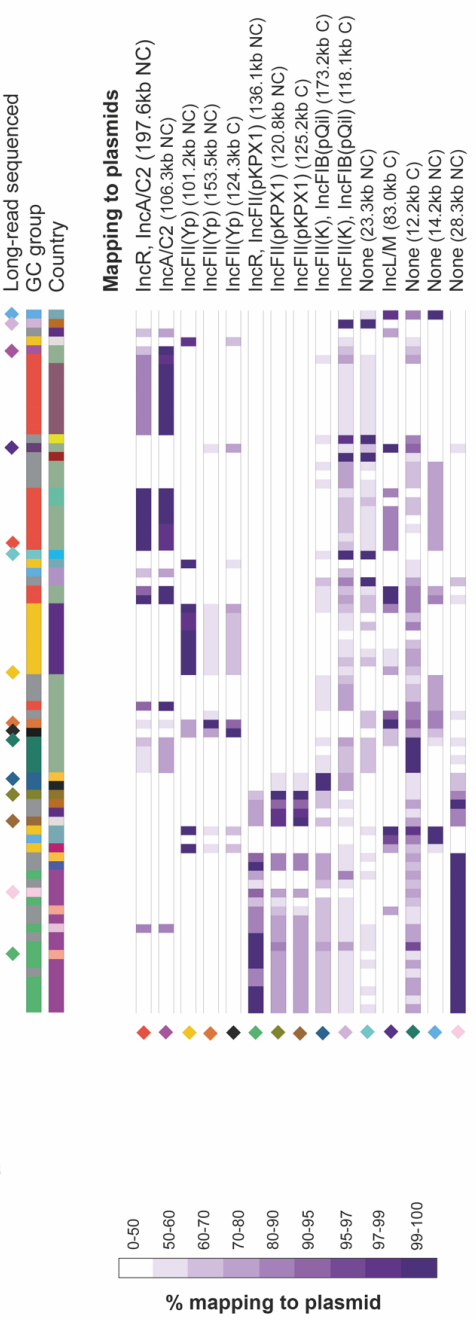

Figure 2. Plasmids carrying bla $\mathrm{VII}_{\mathrm{VI}}$ and $b_{\mathrm{NDM}}$ genes are associated with individual clonal expansions. The phylogenetic trees, constructed using SNPs in the core genome, show 56 bla $\mathrm{VIM}_{\mathrm{M}}$-carrying isolates (A) and 79 bla $_{\mathrm{NDM}}$-carrying isolates (B) from K. pneumoniae sensu stricto. Both trees are midpoint-rooted. We excluded all non-blaviM and non-bla NDM- $_{\text {- }}$ carrying isolates, respectively, which would be interspersed amongst the isolates here. Longread sequenced isolates are marked next to the tree with a diamond if they carry the carbapenemase gene on a putative plasmid sequence or a circle if they carry the gene on the chromosome. The colours of the diamonds represent distinct carbapenemase-carrying plasmids that were obtained. Columns, from left to right, show the genetic context (GC) group 
of isolates assigned using the short-read assembly contigs (ambiguous isolates not assigned to any group are in grey), the country of isolation and the gene variant (for blavim genes only as all bla NDM $_{\text {genes were }}$ bla NDM-1 $_{1}$. Remaining columns show the percentage length of putative plasmids carrying blaviM $(A)$ and bla NDM $(B)$ genes obtained from the hybrid assemblies that were mapped by short reads (note the non-linear colour gradient). The nine reference plasmids used in (A) are from isolates, EuSCAPE_GR073, EuSCAPE_ES094, EuSCAPE_LV006, EuSCAPE_ES220, EuSCAPE_RO094, EuSCAPE_TR203, EuSCAPE_IT062, EuSCAPE_IT312, EuSCAPE_HU009 (left to right in figure). The fifteen used in (B) are from EuSCAPE_RS017, EuSCAPE_RS105, EuSCAPE_TR083, EUSCAPE_RS064, EUSCAPE_RS002, EUSCAPE_PL046, EUSCAPE_CZ007, EUSCAPE_AT023, EuSCAPE_DE019, EUSCAPE_IE008, EuSCAPE_IL075, EuSCAPE_RS010, EuSCAPE_RS081, EuSCAPE_RO052 and EuSCAPE_GR094 (left to right in figure). Each putative plasmid sequence is indicated by a diamond with the same colour as that indicating the isolate in the tree from which the plasmid was recovered. Mapping data for one shorter blavim-carrying putative plasmid is not shown (EuSCAPE_GR075 - 2.9kb).
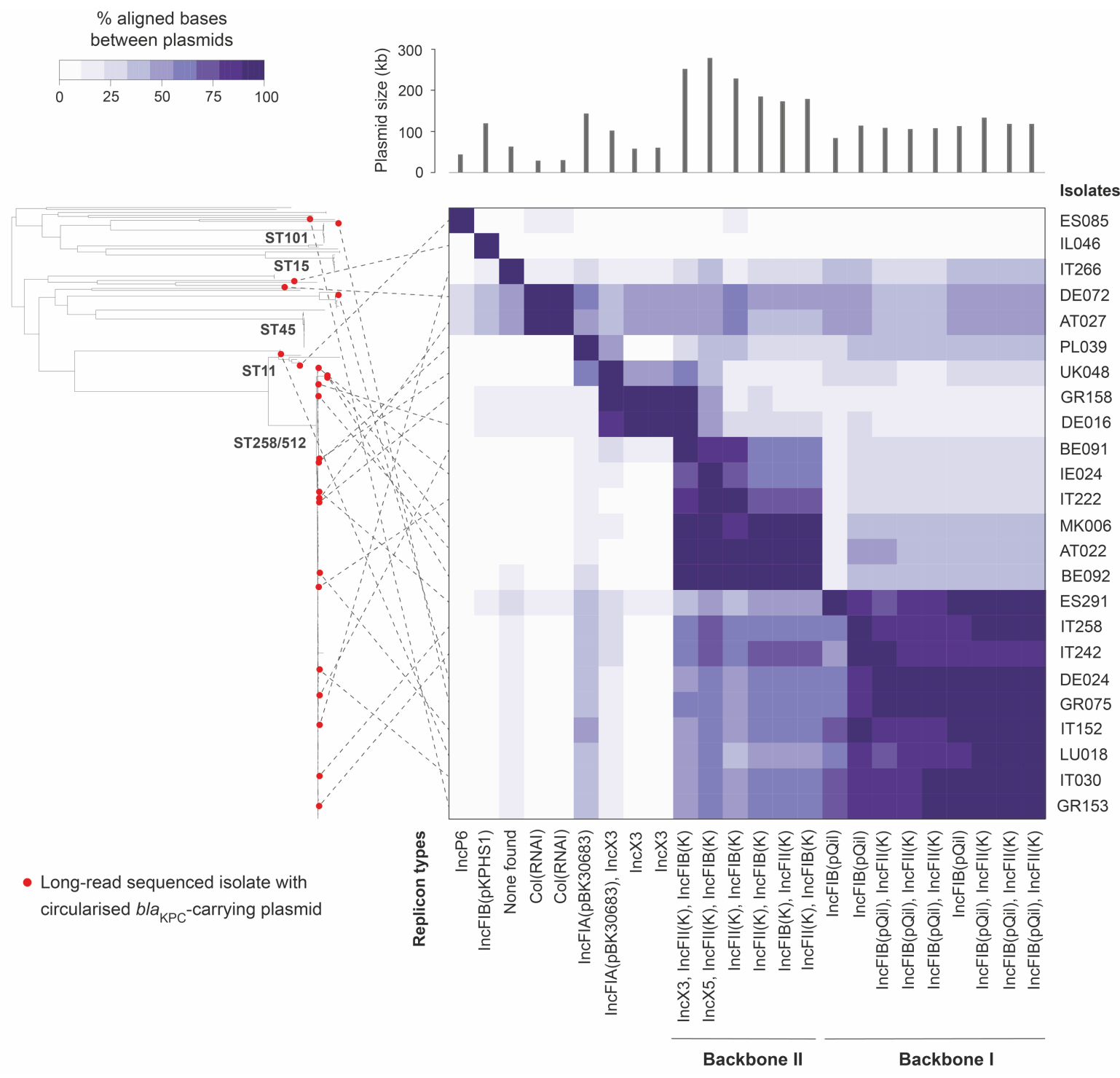
Figure 3. Comparison of 24 circularised bla $a_{\mathrm{KPC}}$-carrying plasmids shows dominance of two major IncF backbone types. The phylogenetic tree contains 311 blakPC-carrying isolates from $K$. pneumoniae sensu stricto (the single bla $\mathrm{KPC}_{\mathrm{C}}$-carrying isolate from $K$. variicola is excluded). The tree was constructed using SNPs in the core genome and is midpoint-rooted. Twenty-four isolates from which circularised blakPC-carrying plasmids were obtained are marked by red circles in the tree. The heat map shows the percentage of bases in each plasmid that could be aligned to each of the other plasmids using NUCmer (the row and column orders are the same). Dotted lines link the 24 long-read sequenced isolates in the phylogenetic tree to their respective plasmids in the heat map. The plasmid length and replicon types found in each plasmid are shown above and below the heat map, respectively.

A

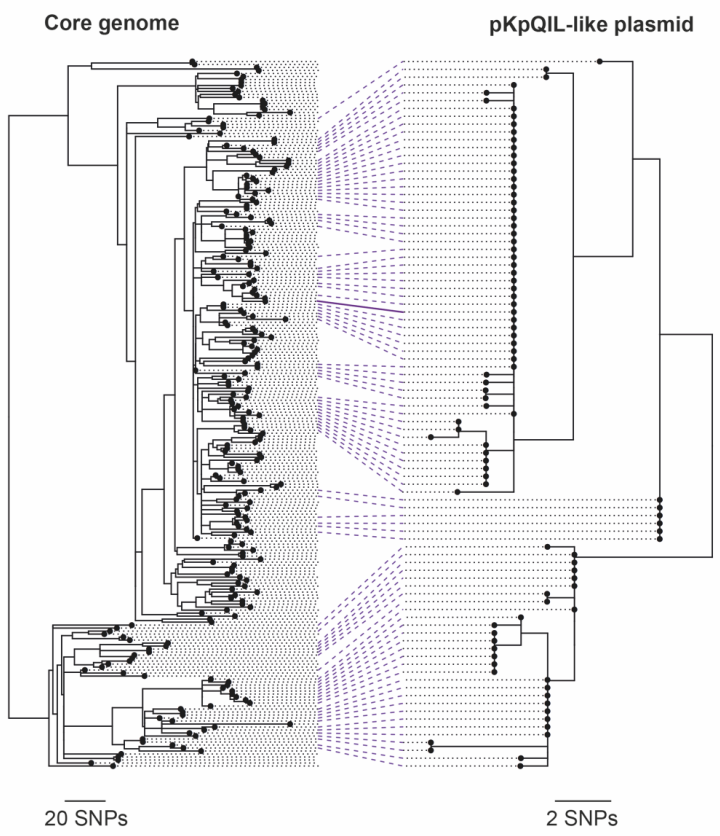

B

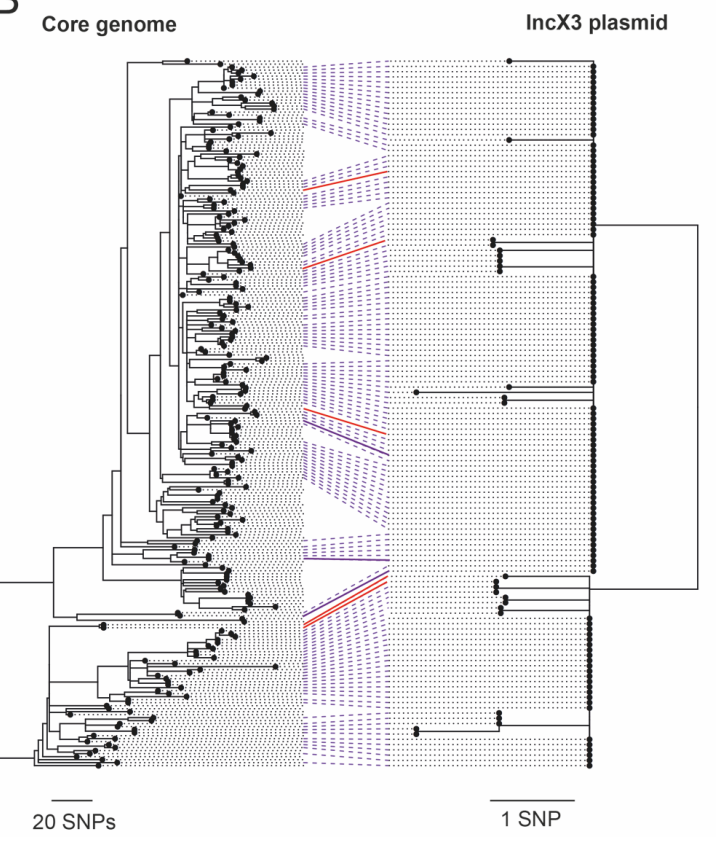

1101

1102

1103

1104

1105

1106

1107

1108

1109

1110

1111

1112

1113

1114

1115

1116

1117

1118

1119

1120
Figure 4. High congruence between pKpQIL-like and IncX3 plasmid phylogenies with the core genome phylogeny of ST258/512 reveals shared evolutionary histories. Each tanglegram comprises a phylogeny of the ST258/512 lineage constructed using all SNPs in the core genome (mapping-based) alignment and either the pKpQIL-like (A) or IncX3 (B) plasmids. The core genome phylogenies include all 236 ST258/512 isolates and were rooted based on previous phylogenetic analyses of the full sample collection that included outgroups of ST258/512 (David et al. 2019). Ninety-one pKpQIL-like and 135 IncX3 plasmid sequences from isolates that had bases $(A / T / C / G)$ called at $\geq 99 \%$ positions in the plasmid reference were included in the plasmid phylogenies. These were rooted to provide the highest concordance with the core genome phylogenies. Lines are drawn between tips in the two trees representing the same isolate. The solid purple lines indicate isolates which were found to carry bla $\mathrm{KPC}$ on a pKpQIL-like (A) or IncX3 (B) plasmid in the hybrid assemblies. Red lines (in (B) only) indicate isolates that were found to carry blakPC on an alternative plasmid to an IncX3 plasmid in the hybrid assemblies. 
A

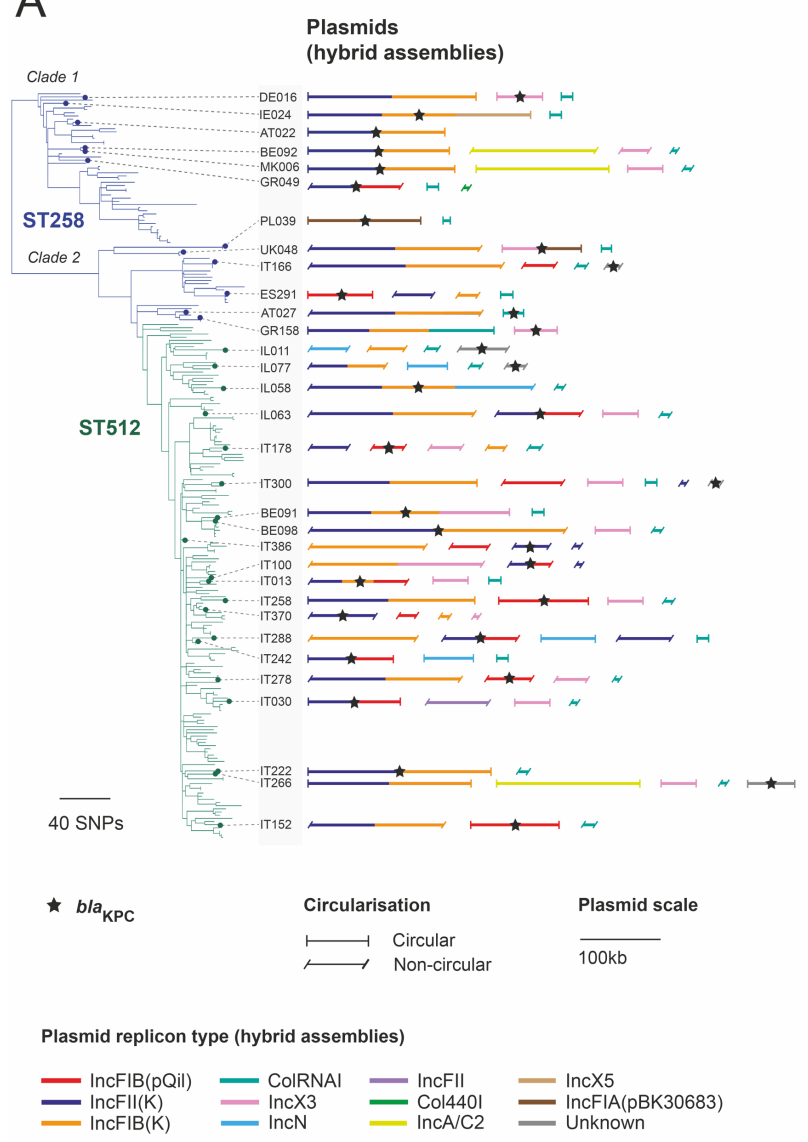

B

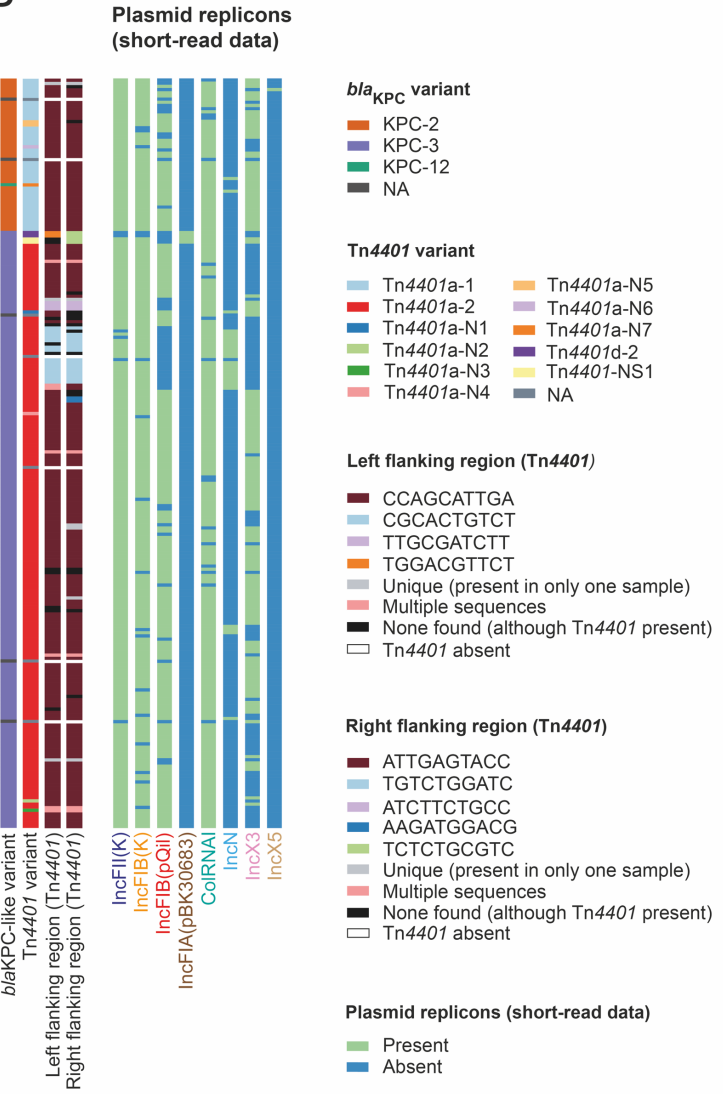

1123

1124

1125

1126

1127

1128

1129

1130

1131

1132

1133

1134

1135

1136

1137
Figure 5. Movement of blakpc genes between plasmids in the ST258/512 lineage. A) The phylogenetic tree contains 236 isolates belonging to ST258/512. It was constructed using SNPs from a core genome (mapping-based) alignment and rooted based on previous phylogenetic analyses of the full sample collection that included outgroups of ST258/512 (David et al. 2019). Thirty-two long-read sequenced isolates carrying blakPC on a putative plasmid sequence are indicated by small circles on the tree tips. Putative plasmid sequences derived from the hybrid genome assemblies with at least one known replicon type and/or containing bla $\mathrm{KPC}_{\mathrm{C}}$ are depicted next to the tree. These are scaled by size and coloured by any replicon types found in the sequence. A star indicates the presence of blakPC within these sequences. B) Metadata columns, from left to right, show the blakPC variant, the Tn4401 variant, the $10 \mathrm{bp}$ left and right flanking regions of Tn4401, and presence or absence of eight plasmid replicon types found using short-read data to be associated with blakPC in the hybrid assemblies. Tn4401a-N1 - Tn4401a-N7 represent novel SNP variants of the structural variant, Tn4401a. Tn4401-NS1 represents a novel structural variant of Tn4401. 